\title{
Erros refrativos e promoção da saúde ocular subsidiada pelo método self-healing
}

Refractive errors and eye health promotion based on the self-healing method

Errores refractivos y promoción de la salud de los ojos subsidiados por el método selfhealing

'João Lucas Barbosa Magalhães ${ }^{1}$, Letícia Santos do Monte ${ }^{1}$, Tatiana do Socorro dos Santos Calandrini ${ }^{1}$, Anneli Mercedes Celis de Cárdenas ${ }^{1}$, Luzilena de Sousa Prudêncio ${ }^{1}$, Silvana Rodrigues da Silva ${ }^{1}$, Nely Dayse Santos da Mata ${ }^{1}$, Rosilda Alves da Silva Isla Chamilco ${ }^{1}$, Rubens Alex de Oliveira Menezes ${ }^{1}$, Maria Virgínia Filgueiras de Assis Mello1*.

\section{RESUMO}

Objetivo: Identificar erros de refração e orientar exercícios visuais subsidiados pelo método self-healing de Meir Schneider para promoção da saúde ocular. Métodos: Estudo de cunho quantitativo e Pequisa-ação com 67 participantes do Projeto Cuide da sua Visão da Universidade Federal do Amapá. Para a operacionalização do estudo os pesquisadores explanavam sobre um determinado erro refrativo e orientavam práticas de exercícios visuais subsidiadas pelo método self-healing. Resultados: Identificou-se prevalência de astigmatismo $(41,79 \%)$, seguido de miopia $(38,8 \%)$, presbiopia $(23,88 \%)$ e hipermetropia $(11,94 \%)$. Quanto aos distúrbios oftalmológicos, o mais prevalente foi a catarata $(11,94 \%)$, estrabismo $(4,47 \%)$, glaucoma $(2,98 \%)$ e visão monocular (2,98\%). Orientou-se exercícios visuais focados no relaxamento profundo, ajuste às frequências luminosas, prática do shifting, olhar longe, expansão da visão periférica e uso equilibrado dos olhos. Conclusão: $O$ método demonstrou sua aplicabilidade para a promoção da saúde ocular à medida que ao implementar os exercícios visuais em seu cotidiano, obteve-se relatos de visão mais nítida, olhos mais lubrificados, diminuição da sensibilidade a luz solar, melhora da fadiga visual e em uma participante com estrabismo, melhora significante do alinhamento ocular. Dada a pouca literatura existente sobre os efeitos mensuráveis dos exercícios visuais e seus benefícios indica-se mais estudos e divulgação deste método.

Palavras-chave: Erros de refração, Autocuidado, Saúde ocular.

\begin{abstract}
Objective: Identify refractive errors and guide visual exercises based on Meir Schneider's self-healing method to eye health promotion. Method: Quantitative study and action research with 67 participants of the "Cuide da sua visão" Project at the Federal University of Amapá. For the operationalization of the study, the researchers explained about a certain refractive error and guided visual exercise practices subsidized by the self-healing method. Results: Prevalence of astigmatism was identified in $41,79 \%$ of the participants, followed by myopia $38.80 \%$, presbyopia $23,88 \%$ and hyperopia $11,94 \%$. As for visual problems, the most prevalent was cataract $8(11,94 \%)$, followed by strabismus $3(4,47 \%)$, glaucoma $2(2,98 \%)$ and monocular vision $2(2,98 \%)$. Visual exercises were oriented focusing on deep relaxation, adjustment to light frequencies, shifting, looking at a distance, expanding peripheral vision and balanced use of the eyes. Conclusion: The method showed its applicability for the eye health promotion, when implementing visual exercises in daily lives, obtained reports of sharper vision, more lubricated eyes, decreased sensitivity to sunlight, improvement of visual fatigue and in strabismus, significant improvement in eye alignment. Given the little existing literature on the measurable effects of visual exercises and their benefits, further studies and dissemination of this method are indicated.
\end{abstract}

Keywords: Refractive errors, Self-care, Eye health.

${ }^{1}$ Universidade Federal do Amapá (UNIFAP), Macapá - AP. *E-mail: virginia@unifap.br 


\section{RESUMEN}

Objetivo: identificar errores de refracción y guiar ejercicios visuales subsidiados por el método de autocuración de Meir Schneider para promover salud ocular. Métodos: estudio cuantitativo e investigación de acción con 67 participantes del proyecto Cuide su visión en la Universidad Federal de Amapá. Para operacionalización del estudio, los investigadores explicaron un cierto error de refracción y prácticas guiadas de ejercicios visuales subsidiados por el método de autocuración. Resultados: se identificó una prevalencia de astigmatismo $(41,79 \%)$, seguida de miopía $(38,8 \%)$, presbicia $(23,88 \%)$ e hipermetropía $(11,94 \%)$. Con respecto a los trastornos oftalmológicos, el más prevalente fue cataratas $(11,94 \%)$, estrabismo $(4,47 \%)$, glaucoma $(2,98 \%)$ y visión monocular $(2,98 \%)$. Los ejercicios visuales orientados, centraron en la relajación profunda, ajuste de frecuencias de luz, shifting, mirar lejos, ampliar la visión periférica y uso equilibrado de los ojos. Conclusión: El método demostró su aplicabilidad para promoción de salud ocular, al implementar ejercicios visuales en vida diaria, hubo informes de visión más nítida, ojos más lubricados, disminución de sensibilidad a luz solar, fatiga visual mejorada y en participante con estrabismo, mejoro significativamente alineación ocular. Dada poca literatura existente sobre efectos medibles de los ejercicios visuales y sus beneficios, se indica una difusión más amplia, como su divulgación.

Palabras clave: Errores de refraccion, Autocuidado, Salud ocular.

\section{INTRODUÇÃO}

Os olhos são órgãos dos sentidos sensíveis e especializados que possuem um sistema óptico para chegada de luz à retina. $O$ olho emétrope é aquele que possui refração normal, resultando em foco claro na retina e ausência de defeitos ópticos, em oposição, o olho amétrope, ao receber a luz não consegue formar a imagem diretamente na retina (HINKLE JL e CHEEVER KH, 2016).

Em todo o mundo, os erros de refração (ER), miopia, hipermetropia, astigmatismo e presbiopia são a principal causa de deficiência visual, e comprometem a visão porque um globo ocular encurtado ou alongado impede que os raios de luz sejam focalizados com nitidez na retina. Em vista disso, os ER constituem problemas oculares comuns que afetam todas as faixas etárias, representando grande desafio à saúde pública (CBO, 2019; SHEELADEVI S, et al., 2019).

Vale acrescentar que a deficiência visual e cegueira causada por ER em adultos pode ter um impacto severo nas questões sociais e bem-estar econômico, incluindo a limitação da educação formal e oportunidades de emprego de pessoas economicamente ativas (NAIDOO KS e JAGGERNATH J, 2012).

A World Health Organization (WHO), estimou que 314 milhões pessoas em todo o mundo, vivem com deficiência visual devido a erros de refração não corrigidos, por conseguinte, os ER foram incluídos como uma das cinco prioridades da OMS na iniciativa global para eliminar a cegueira evitável (WHO, 2017).

Adicionalmente, o Instituto Brasileiro de Geografia e Estatística (IBGE), registra que no Brasil há aproximadamente 7.282 .838 de pessoas com deficiência visual, sendo a maior parte (6.755.664) jovens e adultos entre 18 e 60 anos.

Estima-se que a tendência é que haja um aumento nos percentuais, isto é, mais pessoas com menor acuidade visuais e desenvolvimento de distúrbios visuais e ER, possivelmente decorrentes da influência de fatores como o uso de computadores, celulares e televisão por longos períodos (IBGE, 2013).

No que diz respeito aos ER e demais patologias visuais, este estudo ressalta uma terapia natural complementar, baseada nos escritos do Dr. William Horatio Bates e no método self-healing de Meir Schneider, que se fundamenta na utilização dos olhos de uma forma relaxada e cômoda.

O objetivo principal do método é a promoção da saúde ocular e consiste na prevenção, promoção e recuperação da saúde visual por meio de exercícios compostos por relaxamento visual, massagem, fortalecimento e alongamento ocular (GEBRAEL T, 2019). 
O método self-healing proposto por Meir Schneider tem como um dos focos, a saúde dos olhos como um todo, assim como à prevenção da deterioração da visão relacionada ao estresse visual e a idade. Neste âmbito, Schneider M (2012), propõe a realização de exercícios oculares fundamentais, que quando praticados de forma correta e sistemática atuam preservando uma boa saúde visual.

Nessa perspectiva, considerando que ao exercitar os olhos de maneira adequada, a tendência é que a visão obtenha menor demanda de esforços ao torná-la mais resistente, fundamentados no método selfhealing de mier Schneider, este estudo tem como objetivo identificar erros de refração e orientar exercícios visuais em participantes do "projeto cuida da sua visão" da Universidade Federal do Amapá.

\section{MÉTODOS}

O Projeto cuide da sua visão, iniciou suas atividades em março de 2018 como projeto de extensão e a partir de agosto de 2018 agregou-se a projeto de pesquisa, tendo suas atividades concluídas em outubro de 2019. O projeto era aberto à comunidade e coordenado por duas enfermeiras com a colaboração de acadêmicos.

Para condução deste estudo, elegeu-se a abordagem quantitativa com o emprego do método da pesquisaação, abordagem metodológica na qual os pesquisadores e os participantes representativos da situação ou do problema estão envolvidos de modo cooperativo ou participativo (THIOLLENT M, 2011). A pesquisa também se pauta pela afirmação do mundo da ação, e consequentemente, do fazer, onde o foco do pesquisador embasa-se na compreensão do problema e nas ações para solucioná-lo (NUNES JM e INFANTE M, 1996; FILIPPO D, 2008).

Os encontros do Projeto ocorriam quinzenalmente e os critérios para participação foram: idade mínima de 16 anos e participação com regularidade nos encontros. Durante o período de atividades do Projeto, 67 participantes entre jovens, adultos e idosos, integraram a população do estudo.

Para a operacionalização do estudo, durante os encontros do Projeto ocorriam rodas de conversa sobre a importância dos cuidados com a visão, causas das principais alterações visuais e formas de evitar, explanação sobre a anatomia e funcionamento dos olhos, erros de refração e suas causas, exibição de vídeos e orientações de exercícios específicos para cada tipo de problema visual subsidiados pelo método selfhealing, com ênfase nos seguintes exercícios: Balanço longo: Exercício visual integrativo que melhora o foco e a percepção de luz. Desenvolve fluidez e flexibilidade ocular, possibilitando olhar para os detalhes com maior nitidez porque exercita a mácula, promove a dessensibilização dos olhos a luz solar e estimula a visão periférica; Olhar ao longe: relaxa os músculos ciliares enquanto os ligamentos suspensórios mantêm 0 cristalino pleno e flexível; Estímulo à visão periférica: visa exercitar os bastonetes, células da retina responsáveis pela visão noturna e pela periferia, eliminando grande parte do estresse gerado aos cones, células responsáveis pela visão central; Ensolar: a exposição adequada ao sol diariamente, estiumula a dilatação e contração da pupila, tornando mais forte os músculos da íris. A retina também é favorecida pela luz mais concentrada e a circulação flui melhor em direção ais ilhós em decorrência dos movimentos de contração e expansão da pupila; Passei noturno: exercício complementar ao ensolar, consiste no ato de caminhar durante a noite ou no escuro. Na escuridão bastam três a quatro minutos para que as pupilas se dilatem até nove vezes mais que seu tamanho norma, entretanto para maior eficácia são necessários cerca de 40 minutos para ativar os bastonetes da retina que percebem o movimento e a periferia; Empalmar: auxilia a acalmar a mente e a relaxar os olhos, sendo a prática complementar a todos os demais. Com a prática correta desse exercício ocorre uma descontração nas pálpebras, têmporas e toda região craniana, o aperto dos olhos é eliminado propiciando sensação de relaxamento; Shifting: tem como obetivo ativar a macula e auxiliar no desenvolvimento da retina. Envolve deslocar o olhar para objetos próximos ou contemplar 0 horizonte de detalhe para detalhe, sem lente de contato ou óculos. Essa atividade por sua vez, criam mais sinapses entra a macula e o cérebro e entre o cérebro e a macula; Obstrução do olho dominante: estimula e fortalece o olho mais fraco comparado ao dominante. Quando um dos olhos estiver sendo mais utilizado que o outro, este exercício pode fazer uma grande diferença ao descansar o olho mais forte; Piscar: esse pequeno ato massageia o globo ocular e ativa a umidade e um lacrimejamento natural, provendo vitalidade. $\mathrm{O}$ ato de 
piscar adequadamente influencia automaticamente a sensação de relaxamento e movimento no corpo, além de ajudar no desenvolvimento da visão periférica.

Como instrumento para coleta de dados, utilizou-se uma ficha elaborada pelos pesquisadores contendo variáveis sociodemográficas (idade, sexo, escolaridade e ocupação) e variáveis oftalmológicas (tipo de ER), identificadas a partir da receita de exame oftalmológico do participante. Os ER foram definidos pelo equivalente esférico (EE) e cilíndrico (EC) ametrópicos, miopia com EE $\leq-0.50$ Dioptrias (D), hipermetropia com $E E \geq+0.50 \mathrm{D}$, astigmatismo com $E C \leq-0.5 \mathrm{D}$ e presbiopia com EE para visão de perto $\geq+0.50 \mathrm{D}$.

Durante os encontros também foram realizados testes com a escala de Snellen para medir a acuidade visual. A escala foi aplicada em ambiente bem iluminado, posicionado em uma parede sem janelas a uma distância com cerca de seis metros do participante. A obtenção dos dados iniciou-se em agosto de 2018 e se estendeu durante todo o período de execução do Projeto, sempre que novo participante integrava o grupo.

Os dados foram analisados por meio de tabulação no programa Microsoft Office Excel 2016. Para o tratamento estatístico foi utilizado estatística descritiva através de frequências absolutas e relativas, média e desvio padrão.

O estudo seguiu as diretrizes da Resolução n. 466/2012, do Conselho Nacional de Ética em Pesquisa (CONEP), sendo aprovado pelo Comitê de Ética em Pesquisa da Universidade Federal do Amapá sob Parecer 2.847.200 / CAAE: 96005018.9 .0000 .0003 . A anuência dos participantes do estudo foi documentada mediante a assinatura do Termo de Consentimento Livre e Esclarecido (TCLE).

\section{RESULTADOS}

A partir dos dados obtidos, com relação à idade, observou-se que a faixa etária variou entre 16 e 78 anos, com média e desvio padrão de 39,95 $\pm 17,46$ anos. Em relação ao sexo, $16(23,88 \%)$ participantes eram do sexo masculino e $51(76,11 \%)$ do sexo feminino. Quanto a escolaridade, obteve-se prevalência de participantes com ensino médio completo 37 (55,22\%), seguidos por ensino superior completo $23(34,32 \%)$, ensino fundamental $4(5,97 \%)$ e não alfabetizados $3(4,47 \%)$. No que se refere à ocupação $36(53,73 \%)$ são trabalhadores formais, $27(40,29 \%)$ são estudantes e 4 (5,97\%) aposentados (Tabela 1).

Tabela 1 - Variáveis sociodemográficas da população estudada, n=67. Macapá-AP, 2018/2019.

\begin{tabular}{|c|c|c|}
\hline Faixa etária & (n) & $\overline{(\%)}$ \\
\hline $16-26$ & 29 & 43,28 \\
\hline $27-37$ & 02 & 2,98 \\
\hline $38-48$ & 12 & 17,91 \\
\hline $49-59$ & 13 & 19,40 \\
\hline$>60$ & 11 & 16,41 \\
\hline Média e desvio padrão & \multicolumn{2}{|c|}{$39,95 \pm 17,46$} \\
\hline \multicolumn{3}{|l|}{ Sexo } \\
\hline Masculino & 16 & 23,88 \\
\hline Feminino & 51 & 76,11 \\
\hline \multicolumn{3}{|l|}{ Escolaridade } \\
\hline Não alfabetizados & 3 & 4,47 \\
\hline Ensino fundamental completo & 4 & 5,97 \\
\hline Ensino médio completo & 37 & 55,22 \\
\hline Ensino superior completo & 23 & 34,32 \\
\hline \multicolumn{3}{|l|}{ Ocupação } \\
\hline Estudantes & 27 & 40,29 \\
\hline Trabalhador formal & 36 & 53,73 \\
\hline Aposentados & 4 & 5,97 \\
\hline Total & 67 & 100 \\
\hline
\end{tabular}

Fonte: Magalhães JLB, et al., 2020. 
Em relação às variáveis oftalmológicas, o ER mais prevalente foi o astigmatismo $28(41,79 \%)$, seguido de miopia $26(38,80 \%)$, presbiopia $16(23,88 \%)$ e hipermetropia $8(11,94 \%)$. Quanto aos distúrbios visuais, o mais prevalente foi a catarata $8(11,94 \%)$, seguido de estrabismo $3(4,47 \%)$, glaucoma $2(2,98 \%)$ e visão monocular $2(2,98 \%)$. Observou-se presença de ER associados a outros problemas visuais em 31 participantes (Figura 1).

Figura 1 - Variáveis oftalmológicas da população estudada. Macapá-AP, 2018/2019.

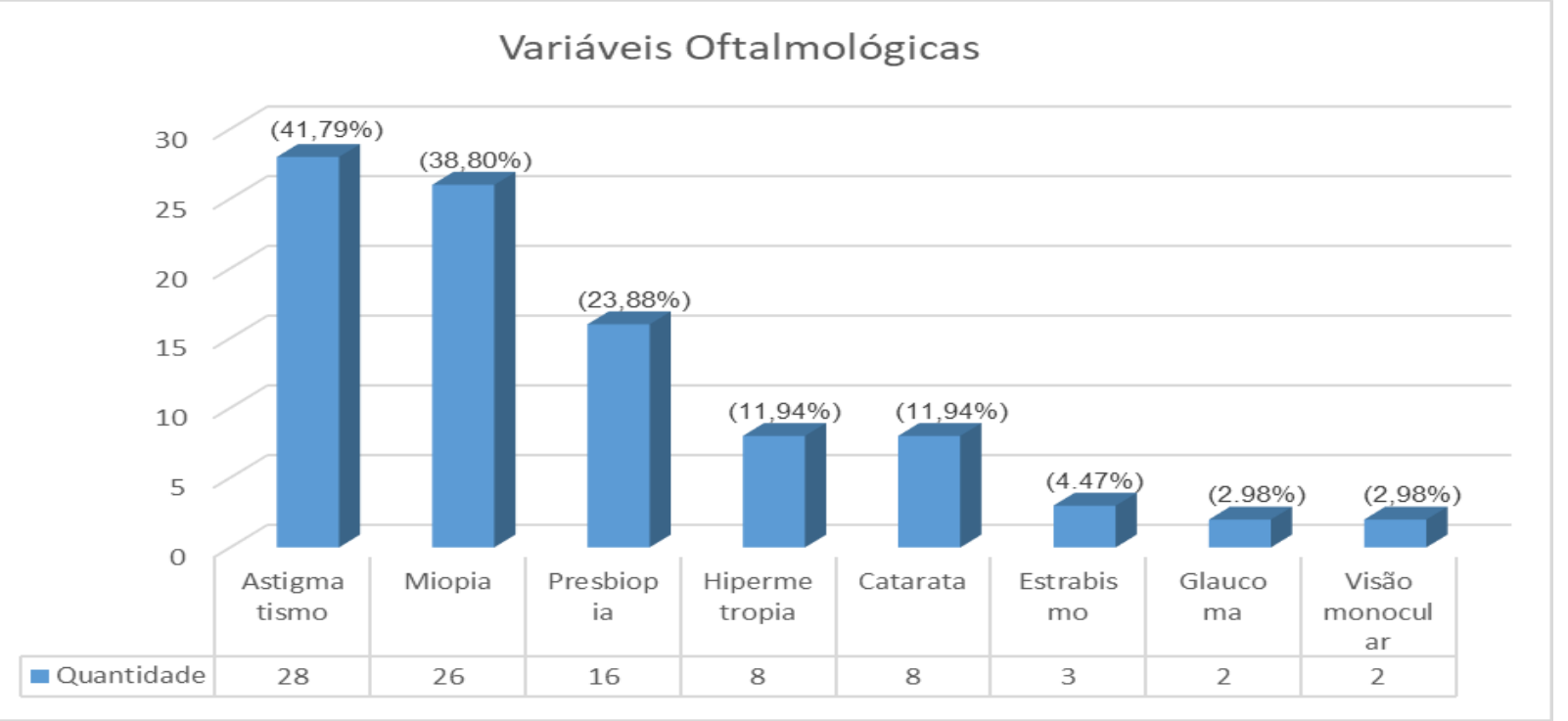

Fonte: Magalhães JLB, et al., 2020.

Mediante a identificação dos ER mais prevalentes, os encontros do Projeto focalizaram na orientação e prática de exercícios específicos em conformidade com o método self-healing direcionados para cada problema visual.

A automassagem, o empalmar e o ensolar, foram exercícios orientados e praticados para todos os ER. No que diz respeito ao astigmatismo, acrescentou-se exercícios com a tabela visual, seguir objetos que brilham no escuro e alongamento dos olhos estático e dinâmico.

Referente à miopia e hipermetropia, implementou-se o shifting por 10' e exercícios de estimulação da visão periférica. Para a presbiopia, foi trabalhado estímulo da visão periférica, olhar para longe e perto durante 10', olhar ao longe durante 20' divididos em duas a três sessões, exercícios de piscar os olhos de forma independente e exercícios de leitura com folha de parágrafos durante 10'. Quanto aos distúrbios patológicos, os exercícios foram orientados e praticados da seguinte forma:

Catarata: exercícios para olhar ao longe 30' ao dia em três intervalos de 10', prática do shifting e tampão no olho mais forte 10' ao dia. Estrabismo: balanço longo 10' ao dia, tampão no olho mais forte 15' por dia, alongamento dos olhos no escuro seguido de alongamento somente do olho estrábico em sentido contrário do desvio, piscar independente cada olho 5' por dia, exercício Melissa 8' por dia e exercícios com óculos verde-vermelho quatro vezes na semana durante 10'.

Glaucoma: estímulo da visão periférica 20' por dia, exercícios com a tabela visual, tampão no olho mais forte e estímulo com leitura do olho mais fraco durante 5'. Visão monocular: prática diária dos exercícios básicos do método, com vistas a preservar a visão do olho funcional.

\section{DISCUSSÃO}

O objetivo do presente estudo foi identificar erros de refração em participantes do Projeto cuide da sua visão da Universidade Federal do Amapá e orientar exercícios visuais subsidiados pelo método self-healing 
de Meir Schneider. Identificou-se o astigmatismo, miopia e presbiopia como os ER mais prevalentes. Estudos conduzidos em âmbito nacional e internacional obtiveram resultados similares (FERRAZ FH, et al., 2015; PAN CW, et al., 2013; WANG M, et al., 2019).

A literatura registra que o astigmatismo resulta da assimetria anterior da córnea, fazendo com que a imagem seja desfocada ao longo de vários pontos da via óptica. Tal ocorrência leva à diminuição da qualidade da imagem, afetando negativamente o desempenho da leitura e a capacidade de dirigir à noite (READ SA, et al., 2014; NARAYANASAMY S, et al., 2015; REYNOLDS ME, et al., 2019).

Estudo com escolares do ensino fundamental em Mato Grosso, identificou entre as ametropias refrativas, o astigmatismo como diagnóstico mais prevalente, isolado ou associado. Tal ocorrência reforça a concepção de que a triagem visual se mostra como uma importante ferramenta para o diagnóstico precoce dos casos de déficit de visão.

Ademais, a prevalência de baixa acuidade visual em escolares justifica a necessidade de incluir a avaliação oftalmológica nas estratégias públicas de saúde ao considerar que quanto mais precoce for o diagnóstico, melhor será o prognóstico dessas crianças (BECKER TOF, et al., 2019).

Ao considerar que a córnea constitui umas das estruturas mais flexíveis do globo ocular, os exercícios visuais propostos pelo método self-healing, atuam aquecendo e tracionando a córnea, tornando-a mais moldável. A prática dos exercícios específicos para astigmatismo, com ênfase no relaxamento e automassagens, o ensolar e alongamento visual, proporcionou melhora do embaçamento visual entre os participantes com o decorrer do tempo.

Nesta mesma perspectiva, Tacca J, et al. (2020) consideram a fisioterapia oftálmica como ferramenta com atuação diferenciada e intervenção capaz de recuperar e/ou prevenir problemas oculares, somando ações à interdisciplinaridade para maior eficácia e eficiência no tratamento da miopia e do astigmatismo.

Morgan IG, et al. (2012), registram que a miopia resulta de um comprimento axial mais longo do olho, fazendo com que as imagens sejam desfocadas na frente da retina e os objetos distantes pareçam embaçados. Entretanto a alta miopia aumenta o risco de doenças oculares patológicas como, catarata, glaucoma, descolamento de retina e degeneração macular miópica, alterações que podem causar perda de visão irreversível (WONG TY, 2014).

A velocidade e a amplitude de expansão da miopia em âmbito populacional surpreenderam a rapidez de respostas de entidades internacionais, à frente a própria OMS e seus comitês de especialistas. Por esta razão, universalizou-se uma agenda para todos os continentes e países, de modo a problematizar as demandas potenciais que desde então se tornaram temas preocupantes e mesmo prioritários de diversas nações do mundo. As evidências são consistentes por si, ao considerar prevalência de miopia em $33 \%$ da população mundial, estando bem caracterizado um problema de saúde pública (WHO, 2015).

Estudo conduzido por Holden BA, et al. (2016), estimou aumento significativo na prevalência global de miopia e alta miopia atingindo 5 bilhões e 1 bilhão de pessoas respectivamente, até 0 ano de 2050. 0 alarmante aumento da miopia e alta miopia são amplamente impulsionados por fatores ambientais, principalmente por mudanças no estilo de vida resultantes de uma combinação entre menor tempo ao ar livre e maior em atividades de trabalho em ambientes fechados, aliado ao uso excessivo de dispositivos eletrônicos a curta distância, causando estresse visual (MORGAN IG, 2012). A predisposição genética também parece desempenhar um papel, mas não pode explicar as tendências temporais observadas em um curto período (LIM LT, et al., 2014).

Ante o exposto e levando em consideração que os movimentos oculares são regidos por músculos, a prática sistemática dos exercícios visuais propostos pelo método self-healing visam regular o tamanho do globo ocular, fortalecer a pupila e o cristalino e proteger a retina. Nesta direção, entre os exercícios implementados para a miopia, deu-se ênfase ao empalmar, ensolar, estímulo da visão periférica, olhar os detalhes, trabalhar o foco para longe e exercícios com a tabela visual. Melhora de a sensibilidade a luz solar e da tensão visual e aumento do foco para longe, foram resultados obtidos a curto e médio prazo entre os participantes do estudo. 
Meningite MC e Taglietti M (2017) destacam que, nos últimos anos as doenças oculares têm se tornado muito comuns em docentes universitários, tendo como fator contribuinte o tempo ampliado em frente às telas de computadores, levando a um aumento gradativo das doenças oculares e fadiga visual e, por isso, medidas preventivas se fazem necessárias, sendo importante adotar hábitos que protejam a saúde dos olhos.

Em vista disso, a prática de exercícios oculares contribuem para a saúde visual, a medida que melhoram o desempenho dos músculos dos olhos, reduzindo o impacto negativo ocasionado pelo estresse visual que se produz na visão diária, evitando cefaleias, promovendo melhora na concentração e em alguns aspectos da visão, proporcionando relaxamento aos músculos dos olhos, além de serem benéficos em uma variedade de doenças oculares, relacionadas ou não com o envelhecimento dos olhos, tais como: miopia, estrabismo, insuficiência de convergência, nistagmo, presbiopia, entre outras (MENINGITE MC e TAGLIETTI M, 2017).

$\mathrm{Na}$ cidade de São Carlos-SP, pesquisadoras elaboram, implementaram e avaliaram um programa de saúde ocular, tendo como base os exercícios do método Self-healing de Meir Schneider em um projeto social destinado à crianças, adolescentes e suas famílias. Ao final do processo de intervenção, ressaltou-se a efetividade da aplicação dos exercícios nas ocasiões de fadiga ocular e dor de cabeça, melhora na acuidade visual, assim como a satisfação pela aquisição de conhecimentos sobre o funcionamento dos olhos (GEBRAEL TLR, et al., 2006).

Estudo realizado no Rio Grande do Sul verificou a influência da Fisioterapia nas alterações visuais de voluntários com astigmatismo e/ou miopia, constatando-se após 24 encontros semanais aumento da acuidade visual, efeitos na presbiopia, na diminuição da fadiga ocular, no desapego do uso dos óculos, aumento da ampliação periférica, na atenção do olhar e aquisição de conhecimento sobre os cuidados com a visão (TACCA J, et al., 2020).

Também Wolff CM e Taglietti M (2019) constataram em seu estudo, que a terapia visual foi eficaz no tratamento da insuficiência de convergência, obtendo-se diminuição significativa dos sintomas, como fadiga ocular, cefaleias, visão turva, diplopia, sonolência, ardor, produção excessiva de lágrimas, dificuldade de concentração, dificuldade de compreensão após curtos períodos de leitura ou após atividades que requerem uso prolongado da visão de perto, além de reduzir o ponto próximo de convergência.

No que diz respeito a presbiopia, popularmente chamada de vista cansada, este ER tem como característica a dificuldade para enxergar de perto. Todavia, a presbiopia é um processo fisiológico que ocorre principalmente por um enrijecimento do cristalino e dos músculos ciliares, resultando em sintomas se manifestam em torno dos 40 anos (SCHNEIDER M, 2012).

Neste estudo a presbiopia esteve presente em 19,40\% dos participantes, corroborando com estudos realizados na Índia e Arábia Saudita (SHEELADEVI S, et al., 2019; JOSEPH S, et al. 2018; PARREY MUR, ELMORSY E, 2019).

Gebrael T (2017), ressalta que os maus hábitos visuais no decorrer da vida tais como não se expor a luz solar, não dormir em ambientes escuros, estresse visual ocasionado por leituras e uso de computador ou celulares por tempo prolongado e sem intervalos para descanso, contribuem para a presbiopia em decorrência da pouca movimentação do cristalino. Nesta perspectiva, os exercícios propostos pelo método self-healing atuam exercitando a contração e relaxamento dos músculos ciliares para tornar o cristalino mais flexível e melhorar o foco para perto.

Em vista disso, exercícios de relaxamento, automassagem, empalmar, alternar olhar longe e perto, movimentos de abrir e fechar a pupila por meio da luz e escuridão proporcionado pelo exercício ensolar, piscar alternadamente, alongamento dos músculos para ajustar a convergência, exercícios para estimular a visão periférica, tampão no olho mais fraco, e exercícios de leitura com folha de parágrafos, foram alguns dos exercícios praticados pelos participantes do Projeto, tendo como resultado melhora da capacidade de leitura.

Ao término das atividades do Projeto Cuide de sua visão, foi ressaltada a importância da continuidade dos exercícios visuais e que estes sejam inseridos em suas atividades cotidianas para melhora progressiva dos benefícios obtidos. Os participantes e pesquisadores avaliaram como positivos os resultados alcançados, ficando evidente a aceitação e interesse dos participantes. 


\section{CONCLUSÃO}

A promoção da saúde ocular através do método self-healing demonstrou sua aplicabilidade para a promoção da saúde por se tratar de terapia natural complementar, considerando que os exercícios propostos se baseiam em técnicas simples, acessíveis e que podem ser implementadas no cotidiano. No decorrer dos encontros realizados no Projeto Cuide da sua Visão, oportunizou-se uma ampla abordagem dos erros refrativos, assim como a orientação e prática de exercícios visuais específicos para os erros de refração: astigmatismo, miopia, hipermetropia e presbiopia. Exercícios para direcionados para catarata, estrabismo e glaucoma, também foram orientados e praticados. Ressalta-se também o fato de ainda serem escassas publicações científicas que abordam efeitos mensuráveis dos exercícios visuais e seus benefícios, indica-se mais estudos e divulgação deste método.

\section{REFERÊNCIAS}

1. BECKER TOF, et al. Avaliação da acuidade visual em escolares do ensino fundamental. Revista Brasileira de oftalmologia, 2019; 78(1): 37-41.

2. BRASIL. Ministério da Saúde. Conselho Nacional de Saúde. Resolução n. 466, de 12 de dezembro de 2012. Diretrizes e normas regulamentadoras de pesquisas envolvendo seres humanos. Diário Oficial da União da República Federativa do Brasil. 2013 jun 13;150 (112 Seção 1): 59-62.

3. CONSELHO BRASILEIRO DE OFTALMOLOGIA. As condições de saúde ocular no Brasil 2019. São Paulo: 2019; $104 p$.

4. FILIPPO D. Suporte à Coordenação em Sistemas Colaborativos: uma pesquisa-ação com aprendizes e mediadores engajados em fóruns de discussão. Tese (Doutorado em Informática) - Pontifícia Universidade Católica-Rio, Rio de Janeiro, 2008; 281p.

5. FERRAZ FH, et al. Refractive errors in a Brazilian population: age and sex distribution. Ophthalmic and Physioloical Optics, 2015; 35:19-27.

6. GEBRAEL TLR. Abra seus olhos: o passo a passo para enxergar melhor sem óculos. ed. BUZZ, 2017; 208p.

7. GEBRAEL TLR, et al. O método Self-Healing e a prevenção em saúde ocular: ações junto a crianças e adolescentes em um projeto social. São Paulo: 2006.

8. HINKLE JL, CHEEVER KH. Brunner e Suddarth: Tratado de Enfermagem Médico-Cirúrgica. 13. ed. Rio de Janeiro: Guanabara Koogan, 2016; 2256p.

9. HOLDEN BA, et al. Global Prevalence of Myopia and High Myopia and Temporal Trends from 2000 through 2050. Ophthalmology, 2016; 123(5): 1036-42.

10. JOSEPH S, et al. Prevalence and risk factors for myopia and other refractive errors in an adult population in southern India. Ophthalmic Physiology, 2018; 38: 346-58.

11. LIM LT, et al. Impact of parental history of myopia on the development of myopia in mainland China school-aged children. Ophthalmology Eye Disease, 2014; 6:31-5.

12. MENIGITE NC, TAGLIETTI M. Sintomas visuais e insuficiência de convergência em docentes universitários. Revista Brasileira de Oftalmologia, 2017; 76(5): 242-246.

13. MORGAN IG, et al. Myopia. The Lancet, 2012; 379(9827): 1739-1749.

14. NAIDOO KS, JAGGERNATH J. Uncorrected refractive errors. Indian Journal of Ophthalmology, 2012; 60(5): 432-437.

15. NARAYANASAMY S, et al. Simulated astigmatism impairs academic-related performance in children. Ophthalmic and Physiological Optics, 2015; 35(1): 8-18

16. NUNES JM, INFANTE M. Pesqisa-Ação: uma metodologia de consultoria. Rio de Janeiro: Editora FIOCRUZ, 1996; $224 p$.

17. PAN CW, et al. Prevalence of refractive errors in a multiethnic Asian population: the Singapore epidemiology of eye disease study. Investigative Ophthalmology \& Visual Science, 2013; 54(4): 2590-2598.

18. PARREY MUR, ELMORSY E. Prevalence and pattern of refractive errors among Saudi adults. Pakistan Journal of Medical Science, 2019; 35(2): 394-398.

19. READ SA, et al. The visual and functional impacts of astigmatism and its clinical management. Ophthalmic and Physiological Optics, 2014; 34(3): 267-294.

20. REYNOLDS ME, et al. Incidence and prevalence of selected refractive errors, active component, U.S. Armed Forces, 2001-2018. Medical Surveillance Monthly Report, 2019; 26(9): 26-30.

21. SISTEMA IBGE. Pesquisa Nacional de Saúde.

22. SCHNEIDER M. Saúde visual por toda a vida. São Paulo: Cultrix, 2012; 328p.

23. SHEELADEVI S, et al. Prevalence of refractive errors, uncorrected refractive error, and presbyopia in adults in India: a systematic review. Indian Jounal of Ophthalmology, 2019; 67(5): 583-592.

24. TACCA J, et al. Práticas fisioterapêuticas na acuidade visual com ênfase na miopia e no astigmatismo. Fisioterapia Brasil, 2020; 21(1): 59-68. 
25. TATIANA GEBRAEL. 2019. In: Bem-vindos ao método self healing. Disponível em: https://metodoselfhealing.com.br/self-healing-bem-vindos/. Acesso em: 22 mar. 2019.

26. THIOLLENT M. Metodologia da Pesquisa-Ação. 18. ed, São Paulo: Cortez, 2011; 136p.

27. WONG TY, et al. Epidemiology and disease burden of pathologic myopia and myopic choroidal neovascularization: an evidence-based systematic review. American Journal of Ophthalmology, 2014; 157(12): 9-25.

28. WOLFF CM, TAGLIETTI M. Exercícios Oculares na Insuficiência de Convergência: Séries de Casos. Oftalmologia, 2019; 42(1): 1-7.

29. WORLD HEALTH ORGANIZATION. Global initiative for the elimination of avoidable blindness: action plan $2006-2011$. Geneva: 2017; 97p.

30. WORLD HEALTH ORGANIZATION. The Impact of Myopia and High Myopia: Report of the Joint World Health Organization-Brien Holden Vision Institute Global Scientific Meeting on Myopia. Sydney: 2015; 32p.

31. WANG M, et al. Prevalence and risk factors of refractive error: a cross-sectional study in Han and Yi adults in Yunnan, China. BMC Ophthalmology, 2019; 19(33): 1-12. 